\title{
Aprendizado do Exame Psíquico por meio de metodologias lúdicas de ensino: Relato
}

\section{de experiência}

\author{
Learning the Psychic Exam through playful teaching methodologies: Experience report \\ Aprender del Examen Psíquico a través de metodologías de enseñanza lúdica: Informe de \\ experiencia
}

\section{Resumo}

Introdução: O exame mental é uma ferramenta essencial para a avaliação psíquica do paciente e a averiguação de possíveis transtornos. Dessa forma, a capacitação e a instrução eficiente dos estudantes de medicina e profissionais de saúde em geral é indispensável, a fim de que o exame mental seja feito corretamente. Nesse sentido, o aprendizado do exame mental por meio de metodologias lúdicas de aprendizagem é viável no ensino da semiologia psiquiátrica. Métodos: realizou-se a metodologia ativa de aprendizagem do tipo sala invertida, isto é, método pelo qual os estudantes adquirem um conhecimento prévio para seus estudos, a partir de uma vivência, seja por meio de um vídeo ou de alguma outra forma de arte (e.g., uma peça teatral); por conseguinte, puderam compartilhar conhecimento entre seus companheiros acadêmicos e professor. Relato de Experiência: expõe-se o ensino do exame psíquico, ludicamente, através de diversos gêneros artísticos, como músicas, poesias, crônicas e peças teatrais. Resultados: os objetivos educacionais foram contemplados adequadamente, tendo os alunos da medicina a capacidade, habilidade e competência não só para transmitir os conhecimentos adquiridos, mas também para aplicá-los na concretização das peças teatrais e mesas-redondas realizadas após as apresentações. Não obstante, os temas a serem trabalhados puderam ser antecipados com os vídeos e músicas, favorecendo assim uma aprendizagem mais significativa. Conclusão: o ensino do exame do estado mental, por meio de metodologias lúdicas, no contexto da semiologia psiquiátrica, favorece uma alternativa eficaz na assimilação do conteúdo e fixação do conhecimento.

Palavras-chave: Aprendizagem; Exame mental; Ludicidade; Psiquiatria; Psicopatologia; Ensino.

\section{Abstract}

Introduction: the mental examination is an essential tool for the psychic assessment of the patient and the investigation of possible disorders. Thus, the training and efficient instruction of medical students and health professionals in general is essential, in order for the mental examination to be done correctly. In this sense, the learning of the mental 
exam through playful learning methodologies is feasible in the teaching of psychiatric semiology. Methods: the flipped classroom-based active learning methodology was used, that is, a method by which students acquire prior knowledge for their studies, from an experience, either through a video or some other art form (eg, a play); therefore, they were able to share knowledge between their academic colleagues and professors. Experience Report: the teaching of the psychic examination is exposed, playfully, through various artistic genres, such as music, poetry, chronicles and plays. Results: the educational objectives were adequately addressed, with medical students having the capacity, skill and competence not only to transmit the acquired knowledge, but also to apply it in the realization of theatrical plays and round tables held after the presentations. Nevertheless, the themes to be worked on could be anticipated with the videos and music, thus favoring a more meaningful learning. Conclusion: the teaching of the examination of the mental state, through playful methodologies, in the context of psychiatric semiology, favors an effective alternative in the assimilation of content and fixation of knowledge.

Keywords: Learning; Mental status examination; Play and playthings; Psychiatry; Psychopathology; Teaching.

\section{Resumen}

Introducción: el examen mental es una herramienta fundamental para la valoración psíquica del paciente y la investigación de posibles trastornos. Por ello, la formación e instrucción eficaz de los estudiantes de medicina y de los profesionales de la salud en general es fundamental para que el examen mental se realice correctamente. En este sentido, el aprendizaje del examen mental a través de metodologías de aprendizaje lúdico es factible en la enseñanza de la semiología psiquiátrica. Métodos: se utilizó la metodología de aprendizaje activo tipo aula invertida, es decir, un método mediante el cual los estudiantes adquieren conocimientos previos para sus estudios, a partir de una experiencia, ya sea a través de un video o alguna otra forma de arte (por ejemplo, una obra de teatro); por lo tanto, pudieron compartir conocimientos entre sus colegas académicos y el profesor. Informe de experiencia: la enseñanza del examen psíquico se expone, de manera lúdica, a través de diversos géneros artísticos, como la música, la poesía, las crónicas y la obra de teatro. Resultados: se abordaron adecuadamente los objetivos educativos, teniendo los estudiantes de medicina la capacidad, destreza y competencia no solo para transmitir los conocimientos adquiridos, sino también para aplicarlos en la realización de obras teatrales y mesas redondas realizadas después de las presentaciones. No obstante, los temas a trabajar se pudieron anticipar con los videos y la música, favoreciendo así un aprendizaje más significativo. Conclusión: la enseñanza del examen del estado mental, a través de metodologías lúdicas, en el contexto de la semiología psiquiátrica, favorece una alternativa eficaz en la asimilación de contenidos y fijación de conocimientos.

Palabras clave: Aprendizaje; Examen mental; Juego e implementos de juego; Psiquiatría; Psicopatología; Ensenãnza.

\section{Introdução}

O exame psíquico é parte fundamental da investigação e entrevista médica (anamnese). Não obstante, as queixas psiquiátricas são comuns na prática clínica diária, e o conhecimento adequado do exame dá subterfúgios para se criar um raciocínio clínico e, assim, auxiliar na tomada de decisão e na opção ou não de encaminhamento adequado. Diante disto, é imprescindível que, sendo especialista ou não, qualquer médico tenha a técnica e o conhecimento adequados para a execução do exame. Dentro desta ótica, a adoção de metodologias lúdicas, no ensino da avaliação mental, é uma alternativa eficiente e viável para o aprendizado dos estudantes e profissionais de saúde em geral (Agra et al., 2019, Gomes et al., 2009).

O ensino dinâmico, participativo e lúdico, o qual se caracteriza a partir de filmes, jogos, brincadeiras, entre outras atividades, faz com que a experiência do aprendizado seja mais interessante para o estudante (Daltro \& Bueno, 2010, DiasLima et al., 2019). Desta forma, com o interesse do conteúdo, o aluno ancora seu conhecimento prévio com o novo (Sousa et al., 2015); além disto, permite que o aluno seja parte ativa na construção de seu conhecimento (Gotardelo et al., 2017). Dentro desta perspectiva, segundo a teoria do aprendizado significativo de David Ausubel, o conhecimento é construído pela associação entre o seu conhecimento prévio (subsunçores) e o novo conhecimento (Agra et al., 2019, Sousa et al., 2015, Tibério et al., 2003, Luna \& Bernardes, 2016). Este conhecimento prévio é exteriorizado a partir de métodos que estimulem a participação do aluno (Sousa et al., 2015), dentre estes, a ludicidade.

De acordo com Moreira (2013), a aprendizagem significativa possui duas características básicas, sendo elas a nãoarbitrariedade e a substantividade. A não-arbitrariedade está relacionada ao uso de conhecimentos prévios relevantes, os quais servirão de pontos de ancoragem. Outrossim, a substantividade está associada à consistência do novo conhecimento, o qual é a ideia ou concepção de um conceito, sendo que pode ser expresso de diferentes maneiras, com diferentes palavras. Assim, o 
ensino lúdico pode servir como uma importante ferramenta no aprendizado, visto que resgata um conhecimento prévio para servir de âncora; e então faz sua junção com o significado de um determinado conhecimento, solidificando-o. Ou seja, os novos conhecimentos serão ancorados de uma forma mais eficaz e o aluno será mais motivado a aprender (Dias-Lima et al., 2019).

Não obstante, conforme a teoria da epistemologia genética de Jean Piaget, o conhecimento é construído a partir da interação entre o sujeito e o objeto de estudo; o estudante incorpora elementos externos em suas estruturas cognitivas, modificando-as (Pontes et al., 2006). Desta maneira, quanto maior o vínculo psicológico e emocional entre o estudante e o conteúdo estudado, maior será a assimilação de conteúdo. Em vista disto, o uso de metodologias lúdicas, além da sua importância na aprendizagem significativa, facilita com que o aluno interaja com o conteúdo a ser aprendido; isto, por consequência, auxilia na absorção de conhecimento e no aprendizado.

Por último, a metodologia lúdica, sob a teoria do desenvolvimento e aprendizagem de Vygotsky, proporciona uma melhor interação entre o mediador e o aluno. O primeiro assiste o estudante a efetivar o desenvolvimento que está próximo; ou seja, facilita a transição entre o desenvolvimento potencial em desenvolvimento real (Cavalcanti, 2005). Isto é concretizado uma vez que o meio lúdico, o qual envolve brincadeiras, jogos, entre outros, possibilita a formação de um ambiente mais desinibido e recreativo entre os participantes.

Neste contexto, este relato de experiência tem como objetivo demonstrar que o exame do estado mental ou exame psíquico pode ser ensinado e aprendido de forma lúdica e o mais significativa possível. Entre estas formas, podem-se apresentá-lo através de músicas, poesias, crônicas e peças teatrais. No tocante aos objetivos educacionais, estabeleceram-se que os alunos fossem capazes de conceituar os temas de cada peça teatral (ex., suicídio, doença de Alzheimer, entre outros); discutir a abordagem, diagnóstico, tratamento e prevenção (ex., suicídio, doença de Alzheimer, entre outros conteúdos programáticos das peças teatrais); aplicar os conhecimentos nos laboratórios de preparação técnica para as peças de teatro (ex.:, hospital psiquiátrico), que ocorreram juntamente com os alunos de artes cênicas envolvidos nos projetos..

\section{Métodos}

O presente trabalho é um estudo qualitativo através de um relato de experiência, cujo conteúdo se baseia em dados obtidos retrospectivamente e descritivamente. Tais achados foram expostos dessa forma, pois, por meio deles, é possível analisar o todo cronologicamente e, ainda assim, levantar os pontos considerados cruciais a fim de discuti-los.

Os relatos de experiência ultrapassam uma identidade meramente descritiva, entretanto também a envolvem. Trata-se de um estudo de natureza qualitativa, relacionado a várias opções teóricas e metodológicas. Um relato de experiência valoriza a explicitação descritiva, interpretativa e compreensiva de diversos fenômenos, dentro de um período histórico (Daltrô \& Farias, 2019).

Para o delineamento da confecção do manuscrito, partiram-se de pressupostos com base nas demandas da prática médica e em teorias psicopedagógicas, para então se trazerem à luz os fatores nos quais as estratégias da sala de aula invertida puderam ser oportunas, enquanto meios facilitadores de aprendizagem. É preciso lembrar que este tipo de aula é uma forma de se trabalhar conteúdos de modo que o sentido da transmissão do conhecimento não se dá primeiramente do professor para os alunos (Bollela, 2017, Ernesto et al., 2018). O que ocorre, porém, nesta sala invertida, é que, num primeiro momento, os discentes terão contato com o conteúdo a ser trabalhado, como, por exemplo, videoaulas, vídeos, podcasts, entre outros (Ernesto et al., 2018). Com esse pré-requisito, seguem para a aula a fim de se aprofundarem com o compartilhamento de conhecimento não só com seus pares, mas também com seu professor. 


\section{Relato de Experiência}

O Exame Psíquico é realizado de forma não-uniforme, visto que cada paciente é único em suas características e aspectos semiológicos (Bastos, 2020). Sendo assim, são abordados os seguintes componentes no Exame psíquico:

1. Apresentação: Atitude: o primeiro aspecto diz respeito à aparência física do paciente, como está sua estética, barba, cabelo, unhas, dentes, adornos, roupas, maquiagem e sua higiene.

A apresentação pode estar adequada, descuidada, bizarra ou exibicionista (caracteriza-se pela excessiva exposição do corpo) e sua postura corporal encurvada, retraída ou inclinada para a frente, o que pode traduzir intensidade de certos estados emocionais. Há fácies típicas que podem ser observadas como fácies melancólicas. Outra questão a ser avaliada é a atitude do paciente com o entrevistador; esta pode ser adequada, colaborativa, hostil, opositora, arrogante, desinibida, teatral, pueril, manipuladora, submissa ou desconfiada.

2. Função psíquica de Consciência: esta pode ser dividida em quantitativa e qualitativa. A primeira se refere ao nível de consciência, que retrata uma capacidade fisiológica do sistema nervoso de se manter em alerta ou com sensório claro.

Quantitativa: Vigil, alerta (normal); hipervigil (aumentada); obnubilado, geralmente causado por ofuscação da visão e obscurecimento da visão; flutuante, com alternância do estado de consciência geralmente associado a quadro orgânico como delirium; comatoso, o qual é um estado de inconsciência em que a pessoa não pode ser despertada.

Qualitativa: Estado crepuscular, caracterizado pelo estreitamento transitório da consciência, com a conservação de uma atividade um tanto coordenada e automática. Normalmente, há falsa aparência de que o paciente está compreendendo a situação; em geral, a percepção do mundo exterior é imperfeita ou de todo inexistente. Dissociação da consciência, que é designado como a fragmentação da consciência, ocorrendo quebra da unidade psíquica como ser humano. Transe ou possessão, o qual se configura como um estado de alteração qualitativa da consciência que se assemelha a sonhar acordado; porém, associado à atividade motora automática e estereotipias acompanhadas de suspensão parcial dos movimentos voluntários.

3. Função psíquica de atenção: refere-se a capacidade de direcionar ou focar a vida mental em determinados estímulos específicos; classifica-se em atenção voluntária (tenacidade), isto é, a capacidade de direcionar, voluntariamente, a vida mental para um estímulo especifico; e a atenção espontânea (vigilância), a capacidade não intencional de redirecionar a vida mental para estímulos novos, a qual é determinada pelas características dos estímulos em questão (por exemplo, intensidade dos mesmos).

Habitualmente, há uma certa relação inversa entre as duas formas de atenção: se a atenção espontânea estiver patologicamente aumentada, a voluntária estará diminuída, e vice-versa. Entretanto, alguns estados cursam com diminuição global de ambas as formas de atenção (hipoprosexia).

4. Função psíquica de orientação: refere-se à capacidade de um indivíduo de conseguir situar-se quanto a si mesmo e quanto ao ambiente. Didaticamente, divide-se a orientação em autopsíquica (identidade do eu) e alopsíquica (quanto ao mundo externo, subdividida em orientação temporal e orientação espacial).

5. Função psíquica de memória: existem diversas classificações e subsistemas de memória. As principais alterações são na fixação, onde pode ser feito o teste das 3 palavras em que consiste falar 3 palavras (por exemplo, leão, sofá e bola) e solicitar que o paciente repita. Para avaliar evocação, solicite que o paciente repita as palavras após uma distração. Outra forma de avaliação é perguntar sobre fatos ocorridos no passado e conferir com algum familiar.

6. Função psíquica de sensopercepção: é a habilidade de captação e codificação de estímulos sensoriais. As distorções na sensopercepção podem ser ilusões ou alucinações; as alucinações são a criação de estímulos inexistentes, e é a que mais interessa numa entrevista clínica; elas podem ser visuais, auditivas, gustativas e motoras. As ilusões são alterações no estímulo recebido e podem ser causadas por diversos fatores como falta de atenção, limitações naturais dos órgãos sensoriais, alterações no sistema de refração, sono, entre outros. As alterações quantitativas da sensopercepção podem ser hiperestesia, hipoestesia e 
parestesias.

7. Função psíquica de pensamento: geralmente, é avaliado com base no discurso do paciente e observa-se a forma, curso e conteúdo. A Forma refere-se a como os pensamentos organizam-se de forma ordenada; pode ser organizada/agregada (lógica), afrouxamento das associações, arboriforme/fuga de ideias, descarrilamento, dissociação e incoerência e desorganizado/desagregado. O curso refere-se à velocidade do pensamento; ele pode ser acelerado, lentificado e bloqueio/roubo de pensamento. O conteúdo refere-se às ideias expressadas pelo paciente; estas podem ser delirantes (crenças irreais não removíveis mediante argumentação lógica), ou possuírem discurso de desvalia, ruína, grandioso, persecutório ou místico-religioso.

8. Funções psíquicas de humor e afeto: denomina-se humor como a disposição de ânimo de uma pessoa em relação a alguma coisa ou em algum momento; em outras palavras, poderíamos dizer que o humor corresponderia à emoção predominante durante a entrevista. $\mathrm{O}$ afeto, por outro lado, designaria a totalidade das emoções no momento em questão, bem como sua relação com o conteúdo do pensamento. Os componentes do afeto são o tônus (quantidade de carga), a modulação (variação) e a ressonância. Seus tipos são labilidade afetiva (passagem súbita de um estado afetivo para outro), dissociação do afeto (incoerência entre a ideia expressa e o afeto presente - La belle indifference), ambivalência afetiva.

9. Função psíquica de psicomotricidade: do ponto de vista quantitativo, a psicomotricidade oscila com a inibição psicomotora, ou abulia e agitação psicomotora. As estereotipias motoras são repetições automáticas e uniformes de determinado ato motor complexo, indicando geralmente marcante perda do controle voluntário sobre a esfera motora. O maneirismo é um tipo de estereotipia motora caracterizada por movimentos bizarros e repetitivos, geralmente complexos, que buscam certo objetivo, mesmo que esdrúxulo. Os tiques são atos coordenados, repetitivos, resultantes de contrações súbitas, breves e intermitentes, envolvendo geralmente um grupo de músculos que atua em suas relações sinérgicas normais; acentuamse muito com a ansiedade.

10. Função psíquica de inteligência: esta deve ser medida não somente por testes de coeficiente de inteligência (QI), mas também através de interpretações de ditados populares como, por exemplo, "Casa de ferreiro, espeto é de pau"; como também, pode-se pedir para o paciente fazer uma abstração relacionada a algum outro ditado popular ou frasão. A pessoa é cada vez mais inteligente quanto às suas condições de adaptação às situações novas e inusitadas.

11. Função psíquica de imaginação: Esta se refere à capacidade de criar e ser original. Entre outros exercícios possíveis, pede-se ao paciente para olhar nas nuvens do céu e descrever o que está vendo; esta imagem é criada em sua mente de acordo com seu estado emocional, recebendo o nome de pareidolia. As fantasias sexuais e de outros conteúdos também fazem parte desta função chamada imaginação.

12. Crítica e noção da doença: Avalia-se o grau de insight do paciente, ou seja, o quanto de compreensão o mesmo apresenta em relação a seu próprio estado mental.

Vale lembrar que tal tipo de exame não segue um modelo fixo, ou seja, para cada paciente, há a escolha de uma forma que se deseja empregar, resultando em variações (Rocha Neto et al., 2019). Elas podem ser entrevistas padronizadas (sejam objetivas ou discursivas) ou semi-padronizadas, sendo cada uma aplicada com o ritmo adequado ao paciente. Assim, a partir dessas diferentes maneiras, é possível acessar os principais sintomas psicopatológicos a fim de delinear o quadro patológico.

Nesse sentido, precisa-se ter em mente que, entre as diversas razões sobre a importância da psicopatologia, está a relação entre a sintomatologia psíquica com a clínica apresentada pelo paciente, já que há uma conexão biográfica sobre como o paciente se porta, sente e expressa com outros transtornos que ele possa apresentar, denotando que os sintomas não são eventos separados, mas são interligados (Stanghellini \& Fiorillo, 2015).

A psicopatologia, frequentemente, é usada como sinônimo para a semiologia psiquiátrica. No entanto, cabe ressaltar que essa se refere, primordialmente, ao contexto envolto de inúmeros fragmentos de experiência, os quais apresentam sentido 
apenas se estiverem inseridos no mesmo contexto (Gorostiza \& Manes, 2011). Desta forma, reforça-se a individualidade de cada transtorno em um paciente, não podendo haver o engessamento do exame psíquico.

Portanto, o exame psíquico tem importância não apenas para o psiquiatra, mas também para o médico generalista, o qual irá se deparar com transtornos psiquiátricos, sendo necessária a individualização de cada paciente quanto ao seu diagnóstico. O exame mental é de extrema relevância também nos serviços de emergências médicas, uma vez que muitos quadros metabólicos e traumáticos podem levar a alterações do nível de consciência e de outras funções mentais. Portanto, é de suma importância que haja um ensino eficaz do exame, o qual permita a retenção do conhecimento a partir da interação entre o médico e conceitos inseridos em um contexto que chame atenção, como peças de teatro, músicas, poesias, entre outras formas lúdicas.

A fim de exemplificar o ensino lúdico do exame psíquico, apresentar-se-ão a seguir os relatos de algumas atividades artísticas que objetivaram transmitir os conhecimentos teóricos sobre o exame mental através de diversas categorias artísticas. Apenas $20 \%$ dos conteúdos didáticos da disciplina foram abordados com o apoio do uso da ludicidade. Para um melhor entendimento, enumerar-se-ão algumas delas:

1. A realização de peças teatrais: Os estudantes de medicina do terceiro ano da Universidade Estadual de Mato Grosso do Sul (UEMS) elaboraram técnica e cientificamente o enredo das peças teatrais (que seguem mais abaixo), nas quais os alunos do curso de artes cênicas da UEMS dirigiram e encenaram.

Nesse caso, os acadêmicos de medicina tiveram a oportunidade de aprimorar suas características de gerenciamento, pois precisaram conciliar seus afazeres de outras disciplinas, com as responsabilidades da confecção e da organização dos atos da peça (e.g., embasamento teórico). Além disso, como trabalharam em grupo, tendo cada participante uma determinada função, puderam trabalhar a questão da gestão de pessoas, bem com a liderança, que são aspectos primordiais, sobretudo tratando-se da prática médica que requer uma desenvoltura não só para se trabalharem em equipes de saúde, mas também para as liderarem (Meireles et al., 2019).

Acrescenta-se ainda que, na elaboração, tais produtores precisaram trazer a teoria para a prática de maneira organizada, coerente, didática e simples; afinal, o público que os iria assistir era amplo, ou seja, a forma como se escreveu a peça precisava ser assimilável por leigos. Tal conjuntura se alinha com as propostas Diretrizes Curriculares Nacionais de 2014 (Meireles et al., 2019), já que, ao aprimorarem predicativos dessa natureza, um maior conhecimento prévio é adquirido para que, quando forem atender pacientes das mais variadas classes e idades, poderão tratá-los mais adequadamente e, por isso, com maior eficácia, adequando a sua postura conforme as situações enfrentadas (Da Silva et al., 2019). Consequentemente, haverá um melhor vínculo médico-paciente, um tratamento mais humanizado e mais empático para sociedade (Mairot et al., 2019).

Ademais, as discussões e temas trazidos nessas encenações são de suma importância. Ao trazer de forma dramática temas cruciais (como o suicídio), ensejou-se uma abertura para que se possa dar relevo a tais assuntos, que nem sempre são discutidos com frequência. Por isso, terminando as encenações, os discentes e docentes abriam um momento para que pudessem ter uma roda de conversa com o público, mostrando assim uma aproximação ainda maior com a sociedade, que ultrapassa as salas da universidade, bem como dos consultórios médicos. Em suma, beneficiam-se os alunos e os docentes, por se aprimorarem e construírem mais conhecimentos; e os membros da sociedade em geral, uma vez que não apenas aprendem um assunto novo e se esclarecem, mas também pelo fato da construção de médicos mais humanizados (Paro \& Silva, 2018).

Cabe ressaltar que algumas dessas peças teatrais receberam premiação de melhor peça teatral estudantil universitária. Podem-se mencionar algumas dessas peças:

1.1. Peça teatral "Nos trilhos da mente: O drama da família e do portador da Demência de Alzheimer" (2017), de autoria do psiquiatra José Carlos Souza. Esta relata a avaliação dos sintomas e sinais da Demência de Alzheimer, tratamento do paciente e dos seus cuidadores. Esta peça foi apresentada apenas três vezes, num intervalo de 15 dias entre elas. As 
apresentações foram em horário extra sala de aula, com 40 minutos de duração.

1.2. Peça teatral "Monólogo sobre o suicídio" (2017), de autoria do psiquiatra José Carlos Souza. Foram discutidas as possíveis causas, abordagem e tratamento dos transtornos mentais que podem estar presentes nas pessoas com ideia, planejamento ou tentativa de suicídio. Destes, 90\% tem algum tipo de transtorno mental, principalmente depressão, ansiedade e envolvimento com drogas lícitas ou ilícitas. Ao final deste monólogo, foi feita uma mesa redonda com a participação de diversos especialistas da área de saúde, para se elucidarem as dúvidas da plateia. É importante se destacar que houve muitos depoimentos de familiares que perderam entes queridos que se suicidaram, o que abalou muito a todos. Esta peça foi apresentada apenas três vezes, num intervalo de 15 dias entre elas. As apresentações foram em horário extra sala de aula, com 30 minutos de duração.

1.3. Peça teatral "Rompimento", de autoria do psiquiatra José Carlos Souza. Essa peça teatral de apenas oito minutos de duração retrata, através de um monólogo, os sinais e sintomas da esquizofrenia, além do seu tratamento. Aborda sinais e sintomas da esquizofrenia e seu tratamento. Esta peça foi apresentada apenas uma vez. A apresentação foi em horário extra sala de aula, com 30 minutos de duração.

1.4. Peça teatral "Teatro sobre a depressão", de autoria do psiquiatra José Carlos Souza. Esta peça discute os sinais, os sintomas e o tratamento em uma mulher jovem e deprimida, a qual teve sua recuperação pelo tratamento integral que ela realizou.

2. A utilização de outros tipos de arte, como a música e a poesia, também foi essencial para se trabalharem temas nas salas de aula. São por meio deles que os alunos tiveram a oportunidade de se obter um conhecimento antecipado, em suas casas, acerca de temas a serem trabalhados, posteriormente, com o docente.

2.1. Apresentação e discussão da música "Eu Nasci Há 10 Mil Anos Atrás" (2018), de autoria de Raul Seixas. No trecho "Eu nasci há 10 mil anos atrás”, encontra-se uma alteração da função psíquica orientação autopsíquica.

2.2. Apresentação e discussão da música "Boate Azul” (2017), de autoria de Benedito Seviero e Aparecido Tomás de Oliveira. Nesta, o trecho "Eu não consigo me lembrar sequer o nome daquela mulher" indica alteração na memória de fixação (fatos recentes), amnésia anterógrada ou de fixação.

2.3. Apresentação e discussão da música "Só Hoje" (2014), de autoria do grupo Jota Quest. O trecho "que eu sou o causador da tua insônia" apresenta uma alteração do sono, a insônia, que é a alteração mais comum relacionada com disfunções mentais e distúrbios de afetividade.

2.4. Apresentação e discussão da música "60 dias Apaixonado" (2018), de autoria de Constantino Mendes e Darci Rossi. No trecho "é num copo que eu me afogo", há uma desorientação alopsíquica quanto ao espaço, uma vez que um corpo não cabe em um copo.

2.5. Apresentação e discussão da poesia "Monólogo de uma sombra" (Sabino, 2005), de autoria de Augusto dos Anjos.

E foi então para isto que esse doudo

Estragou o vibrátil plasma todo,

À guisa de um faquir, pelos cenóbios?!...

Num suicídio graduado, consumir-se,

E após tantas vigílias, reduzir-se

À herança miserável de micróbios! 
Exemplifica-se a ideia de suicídio e tentativa. Percebe-se também uma alteração do instinto de vida e de morte, dentro da função psíquica da afetividade.

2.6. Apresentação e discussão da música " 8 o andar" (2019), de autoria de Clarice Falcão.

E aí, só nós dois no chão frio

De conchinha bem no meio fio

No asfalto riscado de giz

Imagina que cena feliz.

Este trecho é um caso de suicídio duplo, alteração da afetividade no item instinto de vida e de morte. Esta é semelhante à peça Hamlet de William Shakespeare, a qual também apresenta um caso de suicídio duplo.

2.7. Apresentação e discussão da música “Anjos de Deus” (2019), de autoria de Elizeu Gomes.

Tem anjos voando neste lugar

No meio do povo e em cima do altar.

Subindo e descendo em todas as direções.

O trecho mostra a alteração qualitativa na sensopercepção, chamada alucinação visual. Sendo que nesta, a pessoa tem uma imagem perceptiva sem o objeto e tem uma convicção de que está vendo, independente do contexto.

2.8. Visualização e discussão de trecho do programa televisivo "Escolinha do Professor Raimundo" (1988), criação de Chico Anísio. Um de seus personagens de nome Rolando Lero apresenta uma alteração do pensamento denominada tangencialidade. Nesta alteração, o "aluno" conta várias histórias, mas não chega a uma resposta ao que lhe foi perguntado.

Estas formas de aprendizado foram muito elogiadas pelos alunos, com ótimos resultados em suas avaliações cognitivas e formativas. Comprovaram-se que, quanto mais lúdico e significativo for apresentado o conteúdo, melhor será o processo de aprendizagem.

\section{Conclusão}

O exame do estado mental continua sendo o melhor instrumento de avaliação das funções mentais, em quaisquer especialidades clínicas e psiquiátricas; cabe ressaltar que este pode detectar problemas sérios de oscilações dos níveis de consciência, entre outras alterações, nos diversos tipos de serviços de atendimento médico. Em virtude de ser composto de vários conceitos e vocábulos, uma forma lúdica, artística e significativa deve ser requerida para a assimilação cada vez maior de seu conteúdo temático. A ludicidade é uma propulsora da atenção e da motivação dos estudantes, mobilizando suas capacidades cognitivas e criativas, tornando assim a aprendizagem o mais amplo e significativa possível.

\section{Referências}

Agra, G., Formiga, N. S., Oliveira, P. S. D., Costa, M. M. L., Fernandes, M. D. G. M., \& Nóbrega, M. M. L. D. (2019). Analysis of the concept of Meaningful Learning in light of the Ausubel's Theory. Revista Brasileira de Enfermagem, 72(1), 248-55. https://doi.org/10.1590/0034-7167-2017-0691

Anjos de Deus. (2019). Youtube. Vídeo: 4 min. https://www.youtube.com/watch?v=0nBJRvI1Pvw

Bastos, C. L. (2020). Manual do exame psíquico: Uma introdução prática à psicopatologia. Thieme Revinter.

Boate Azul, de Benedito Seviero e Aparecido Tomas, com José e Nico. (2017). Youtube. Vídeo: 3 min. https://www.youtube.com/watch?v=eLjkRfG5yyM

Bollela, V. R. (2017). Sala de aula invertida na educação para as profissões de saúde: Conceitos essenciais para a prática. Revista Eletrônica de Farmácia, 14(1), 39-48. https://www.revistas.ufg.br/REF/article/view/42807

Cavalcanti, L. S. (2005). Cotidiano, mediação pedagógica e formação de conceitos: Uma contribuição de Vygotsky ao ensino de geografia. Cadernos CEDES, 25(66), 85-207. https://doi.org/10.1590/S0101-32622005000200004 
Da Silva, S. R., Da Silveira, L. G., Fraga, L. P., \& Gomes, O. V. (2019). A dramatização como estratégia de ensino-aprendizagem na perspectiva discente: Um relato de experiência no curso de medicina. Revista de Medicina, 98(5), 324-328. https://www.revistas.usp.br/revistadc/article/view/158998

Daltro, M. R., \& Bueno, G. (2010). Ludicidade e narrativa: estratégias de humanização na graduação médica. Revista Brasileira de Educação Médica, 34(4), 497-505. https://doi.org/10.1590/S0100-55022010000400004

Daltro, M. R. \& Faria, A. A. (2019). Relato de experiência: Uma narrativa científica na pós-modernidade. Estudos e Pesquisas em Psicologia, 19(1), 223-237. http://pepsic.bvsalud.org/scielo.php?script=sci_arttext\&pid=S1808-42812019000100013\&lng=pt\&tlng=pt.

Dias-Lima, A., Silva, M. D. C., Ribeiro, L. C. V., Bendicho, M. T., Guedes, H. T. V., \& Lemaire, D. C. (2019). Avaliação, ensinagem e metodologias ativas: uma experiência vivenciada no componente curricular Mecanismos de Agressão e de Defesa, no curso de Medicina da Universidade do Estado da Bahia, Brasil. Revista Brasileira de Educação Médica, 43(2), 216-224. https://doi.org/10.1590/1981-52712015v43n2rb20180037

Ernesto, T. S., Souza, C. H. M., \& Freitas, A. C. A. (2018). Prática de sala de aula invertida: Estrutura didática e avaliativa da aprendizagem na disciplina de fundamentos didáticos. Inter Science Place, 14(2), 163-182. http://www.interscienceplace.org/isp/index.php/isp/article/view/831/486

Escolinha do Professor Raimundo | Rolando Lero. (1988). Youtube. 2020. Vídeo: 4 min. https://www.youtube.com/watch?v=kgWi_NlvBB8

Eu Nasci Há 10 Mil Anos Atrás. (2018). Youtube. Vídeo: 5 min. https://www.youtube.com/watch?v=fHwCCffKKmk

Gomes, A. P., Rôças, G., Coelho, U. C. D., Cavalheiro, P. O., Gonçalvez, C. A. N., \& Siqueira-Batista R. (2009). Ensino de ciências: Dialogando com David Ausubel. Revista Ciências \& Ideias, 1(1), 23-31.

Gorostiza, P. R., \& Manes, J. A. (2011). Misunderstanding Psychopathology as Medical Semiology: An Epistemological Enquiry. Psychopathology, 44(4), 205-215. https://doi.org/10.1159/00032269

Gotardelo, D. R., Bollela, V. R., Souza, A. P. G., Barros, D. D. P., Balbino, J. M. V., \& Ballester, D. (2017). Role-Play Preceded by Fieldwork in the Teaching of Pharmacology: From "Raw Sap" to "Elaborated Sap". Revista Brasileira de Educação Médica, 41(3), 533-539. https://doi.org/10.1590/1981$52712015 v 41 n 3 R B 20160043$

Jota Quest - Só Hoje. (2014). Youtube. Vídeo: 4 min. https://www.youtube.com/watch?v=TynFsTZlGDU

Luna, W. F., \& Bernardes, J. S. (2016). Tutoria como Estratégia para Aprendizagem Significativa do Estudante de Medicina. Revista Brasileira de Educação Médica, 40(4), 653-662. https://doi.org/10.1590/1981-52712015v40n4e01042015

Mairot, L. T. D. S., Costa, B. B. G. D., Heringer, T. P. M., Borges, R. C., \& Moura, E. P. (2019). As Artes na Educação Médica: Revisão Sistemática da Literatura. Revista Brasileira de Educação Médica, 43(4), 54-64. https://doi.org/10.1590/1981-52712015v43n4rb20180146

Meireles, M. A. C., Fernandes, C. C. P., \& Silva, L. S. (2019). Novas diretrizes curriculares nacionais e a formação médica: expectativas dos discentes do primeiro ano do curso de Medicina de uma instituição de ensino superior. Revista Brasileira de Educação Médica, 43(2), 67-78. https://doi.org/10.1590/1981$52712015 \mathrm{v} 43 \mathrm{n} 2 \mathrm{rb} 20180178$

Monólogo sobre o Suicídio. (2017). Youtube. Vídeo: $22 \mathrm{~min}$. https://www.youtube.com/watch?v=f3_rvXmGf1c

Moreira, M. A. (2013). Aprendizagem significativa, organizadores prévios, mapas conceituais, diagramas e unidades de ensino potencialmente significativas. Porto Alegre: Instituto de Física da UFRGS, pp. 1-24.

Nos Trilhos da Mente - Peça Teatral sobre Alzheimer. (2017). Youtube. Vídeo: 47 min. https://www.youtube.com/watch?v=XwJJ_CzPtAE

Oitavo Andar - Clarice Falcão (Legendado). Youtube. (2019). Vídeo: 2 min. https://www.youtube.com/watch?v=G12s1atHo5Q

Paro, C. A., \& Silva, N. E. K. (2018). Teatro do oprimido e promoção da saúde: Tecendo diálogos. Trabalho, Educação e Saúde, 16(2), 471-493. https://doi.org/10.1590/1981-7746-sol00110

Pontes, A. L., Rego, S., \& Silva Junior, A. G. D. (2006). Saber e prática docente na transformação do ensino médico. Revista Brasileira de Educação Médica, 30(2), 66-75. https://doi.org/ https://doi.org/10.1590/S0100-55022006000200009

Rocha Neto, H. G., Estellita-Lins, C. E., Lessa, J. L. M., \& Cavalcanti, M. T. (2019). Mental State Examination and Its Procedures - Narrative Review of Brazilian Descriptive Psychopathology. Front Psychiatry, 10, 77. https://doi.org/10.3389/fpsyt.2019.00077

Sabino, M. P. (2005). O positivismo na poesia de Augusto dos Anjos. Revista Gatilho, 1.

Sessenta dias apaixonado de Darcy Rossi e Constantino Mendes, com Regis e Robson. (2018). Youtube. Vídeo: 2 min. https://www.youtube.com/watch?v=4czTShlf5EU

Sousa, A. T. O., Formiga, N. S., Oliveira, S. H. S., Costa, M. M. L., \& Soares, M. J. G. O. (2015). A utilização da teoria da aprendizagem significativa no ensino da Enfermagem. Revista Brasileira de Enfermagem, 68(4), 713-722. https://doi.org/10.1590/0034-7167.2015680420i

Stanghellini, G., \& Fiorillo, A. (2015). Five reasons for teaching psychopathology. World Psychiatry, 14(1), 107-108. https://doi.org/10.1002/wps.20200

Tibério, I., Atta, J., \& Lichtenstein, A. (2003). O aprendizado baseado em problemas - PBL. Revista de Medicina, 82(1-4), 78-80. 